\title{
Nomogram model for predicting cause- specific mortality in patients with stage I small-cell lung cancer: a competing risk analysis
}

Jianjie $\mathrm{Li}^{{ }^{1 \dagger}}$, Qiwen Zheng ${ }^{2 \dagger}$, Xinghui Zhao ${ }^{1 \dagger}$, Jun Zhao ${ }^{1}$, Tongtong $A n^{1}$, Meina Wu${ }^{1}$, Yuyan Wang ${ }^{1}$, Minglei Zhuo ${ }^{1}$, Jia Zhong ${ }^{1}$, Xue Yang ${ }^{1}$, Bo Jia ${ }^{1}$, Hanxiao Chen ${ }^{1}$, Zhi Dong ${ }^{1}$, Jingjing Wang ${ }^{1}$, Yujia Chi ${ }^{1}$, Xiaoyu Zhai ${ }^{1}$ and Ziping Wang ${ }^{1 *}$

\begin{abstract}
Background: The five-year cumulative incidence rate in patients diagnosed with stage I small-cell lung cancer (SCLC) who were instructed to undergo surgery was from 40 to 60\%.The death competition influence the accuracy of the classical survival analyses. The aim of the study is to investigate the mortality of stage I small-cell lung cancer (SCLC) patients in the presence of competing risks according to a proportional hazards model, and to establish a competing risk nomogram to predict probabilities of both cause-specific death and death resulting from other causes.

Methods: The study subjects were patients diagnosed with stage I SCLC according to ICD-O-3. First, the cumulative incidence functions (CIFs) of cause-specific death, as well as of death resulting from other causes, were calculated. Then, a proportional hazards model for the sub-distribution of competing risks and a monogram were constructed to evaluate the probability of mortality in stage I SCLC patients.

Results: 1811 patients were included in this study. The five-year probabilities of death due to specific causes and other causes were 61.5 and 13.6\%, respectively. Tumor size, extent of tumor, surgery, and radiotherapy were identified as the predictors of death resulting from specific causes in stage I SCLC. The results showed that surgery could effectively reduce the cancer-specific death, and the one-year cumulative incidence dropped from 34.5 to 11.2\%. Like surgery, chemotherapy and radiotherapy improved the one-year survival rate.

(Continued on next page)
\end{abstract}

\footnotetext{
* Correspondence: wangzp2007@126.com

${ }^{\dagger}$ Jianjie Li, Qiwen Zheng and Xinghui Zhao contributed equally to this work and should be considered co-first authors.

${ }^{1}$ Key Laboratory of Carcinogenesis and Translational Research (Ministry of Education/Beijing), Department of Thoracic Medical Oncology, Peking University Cancer Hospital \& Institute, 52 Fucheng Road, Haidian District, Beijing 100142, China

Full list of author information is available at the end of the article
}

(C) The Author(s). 2020 Open Access This article is licensed under a Creative Commons Attribution 4.0 International License, which permits use, sharing, adaptation, distribution and reproduction in any medium or format, as long as you give appropriate credit to the original author(s) and the source, provide a link to the Creative Commons licence, and indicate if changes were made. The images or other third party material in this article are included in the article's Creative Commons licence, unless indicated otherwise in a credit line to the material. If material is not included in the article's Creative Commons licence and your intended use is not permitted by statutory regulation or exceeds the permitted use, you will need to obtain permission directly from the copyright holder. To view a copy of this licence, visit http://creativecommons.org/licenses/by/4.0/ The Creative Commons Public Domain Dedication waiver (http://creativecommons.org/publicdomain/zero/1.0/) applies to the data made available in this article, unless otherwise stated in a credit line to the data. 
(Continued from previous page)

Conclusions: We constructed a predictive model for stage I SCLC using the data from the SEER database. The proportional sub-distribution models of competing risks revealed the predictors of death resulting from both specific causes and other causes. The competing risk nomogram that we built to predict the prognosis showed good reliability and could provide beneficial and individualized predictive information for stage I SCLC patients.

Keywords: SCLC, Competing risks, Cumulative incidence, Nomogram

\section{Background}

Small-cell lung cancer (SCLC) is one of the two main types of lung cancer with short doubling time, high malignancy, and early and extensive metastasis, accounting for approximately $15 \%$ of the lung malignancies. SCLC is sensitive to radiotherapy and chemotherapy but highly prone to drug resistance and relapse. The incidence of SCLC is 6.0 per1000,000 persons [1], and the five-year survival rate is $7 \%$. Because of the pathophysiological characteristics of SCLC, a vast majority of patients have been diagnosed with lymph nodes or distant metastases and lost indications for surgical treatment. Patients with stage I SCLC were recommended to take surgery and postoperative chemotherapy according to National Comprehensive Cancer Network (NCCN) Clinical Practice Guidelines in Oncology (version 2.2018) [2].

Survival analyses are common statistical analysis methods in prognosis research; however, classical survival analyses generally deal with only one type of event, which the researchers are interested in, for example, relapse. Many SCLC patients ultimately die from other diseases instead of lung cancer, indicating that there are death competition causes in SCLC; therefore, it is necessary to use a competing risk regression model when evaluating the prognosis of SCLC. In the presence of competing risks, the classical survival analyses are inaccurate because we cannot assume that the follow-up period is sufficiently long for the event we care about to occur. Nomograms are statistical models, and the basic principle of nomograms is to provide the score of each influencing factor according to the contribution degree of each influencing factor in the regression model, and then, calculate the total score of an individual, so as to obtain the predicted value of the individual.

In this study, we aimed to evaluate the effects of the competing causes for the SCLC survival rate and to establish a competing risk nomogram to quantitatively analyze the survival differences in SCLC patients.

\section{Methods}

\section{Study population}

The data on patients with stage IA and IB small cell lung cancer (SCLC) were obtained from the SEER database (2004-2014) using SEER*Stat (v8.3.2). The study cohort consisted of the patients with the following International
Classification of Diseases for Oncology Third Edition (ICD-O-3), morphology codes: 8002/3; 8041/3, 8042/3, $8043 / 3,8044 / 3$, and 8045/3; and the site codes: C34.0, C34.1, C34.2, C34.3, C34.8, and C34.9. The exclusion criteria were as follows: (1) age at diagnosis less than 18 years, (2) dead or without pathological information, and (3) lack of complete epidemiology and clinical information.

The demographic and clinical pathological data included age, gender, race, anatomical site, laterality, tumor size, tumor degree, grade, and treatment forms. Race was divided into black, white, and others. Three groups were formed according to age (less than 60 years, 60-75 years, and more than 75 years). The anatomic sites were divided into upper, middle, lower, bronchus, and others. Laterality included left and right. The extent of tumor was divided into local and regional, and the grading was classified as good, moderate, poor, undifferentiated, and NOS. The forms of treatment were surgery, chemotherapy, and radiotherapy. The complete SEER session information was added to a supplemental document.

\section{Statistical analysis}

The primary end-point of the study was cause-specific mortality. According to the cause of death (COD) code, we classified the cause of death as cancer-specific death and death resulting from other causes. The covariates added to the model were mainly selected from the available clinically prognostic factors recorded in the SEER database. The covariates included were gender, age, race (black, white, or others/unknown), anatomic sites (upper, middle, lower, bronchus, or others), laterality (left or right), tumor size, extent of tumor (local or regional), grading (good, moderate, poor, undifferentiated, or NOS), chemotherapy (yes or no), radiotherapy (yes or no), and surgery (yes or no). For describing the probability of death, we chose the cumulative incidence function (CIF) and Gray's test [3]. Ages at diagnosis were regrouped as follows: less than 60 years, 60-75 years, and more than 75 . Tumor sizes were grouped into three categories: $\leq 3 \mathrm{~cm}, 3-5 \mathrm{~cm}$, and $>5 \mathrm{~cm}$.

We adopted the Fine and Gray proportional hazards model to assess the three- and five-year probabilities of the two competing mortality events [4]. The restricted 
cubic splines with three empirical knots (10, 50, and 90\%) were fitted to the model [5]. Gray's test was used to compare the difference in the CIF between the two different outcomes. Backward stepwise selection based on Bayesian Information Criterion was used to further eliminate redundant variables. The resulting multivariate Cox regression model was used to calculate risk score and build the final nomogram prognostic model. The Harrell $\mathrm{C}$ index 5 was applied to indicate the discrimination, and the calibration plot obtained using the method provided by Gray [3] was adopted to evaluate the calibration $[6,7]$. Both discrimination and calibration were assessed by bootstrapping with 1000 resamples.

All the statistical analyses were carried out with the $R$ software (v3.3.3). The $\mathrm{R}$ packages cmprsk [8], mstate [9] and rms [10] were used for modeling and developing the nomogram. All the reported significance levels were two-sided, and the $P$ value for statistical significance was defined as $P<0.05$.

\section{Results}

\section{Patient characteristics}

We selected 1811 eligible stage I SCLC patients (Fig. 1). The distribution of the patients' demographics and clinical characteristics is presented in Table 1. Of these, $342(18.9 \%)$ patients were aged $<60$ years, $981(54.2 \%)$ were aged $60-74$ years, and $488(26.9 \%)$ were aged more than 75 years. The number of female patients was 949 (52.4\%) and that of the Caucasians was 1578 (87.1\%). The most common site was the upper lobe (56.6\%), followed by the lower lobe (27.9\%) and the other areas $(15.56 \%)$. The number of patients with a right-sided primary tumor was $1018(56.2 \%)$. The distribution of the tumor size was $53.4,28.9$, and $17.7 \%$ for $<3 \mathrm{~cm}, 3-5 \mathrm{~cm}$, and $>5 \mathrm{~cm}$. As for the tumor extension, the local and the regional ones accounted for 84.3 and $15.7 \%$, respectively. In all, 457 (25.2\%) patients were treated with surgery, 929 (51.3\%) patients were treated with radiotherapy, and 1217 (67.2\%) patients were treated with chemotherapy.

The median follow-up for these patients was 16 months (range: 7 to 33 months). During the followup period, 1221 patients died: 986 died of specific causes, and 235 died of other causes. The top three other causes of death were heart disease (27.2\%), chronic obstructive pulmonary disease (COPD) and allied conditions (20.9\%), and cerebrovascular diseases (4.7\%).

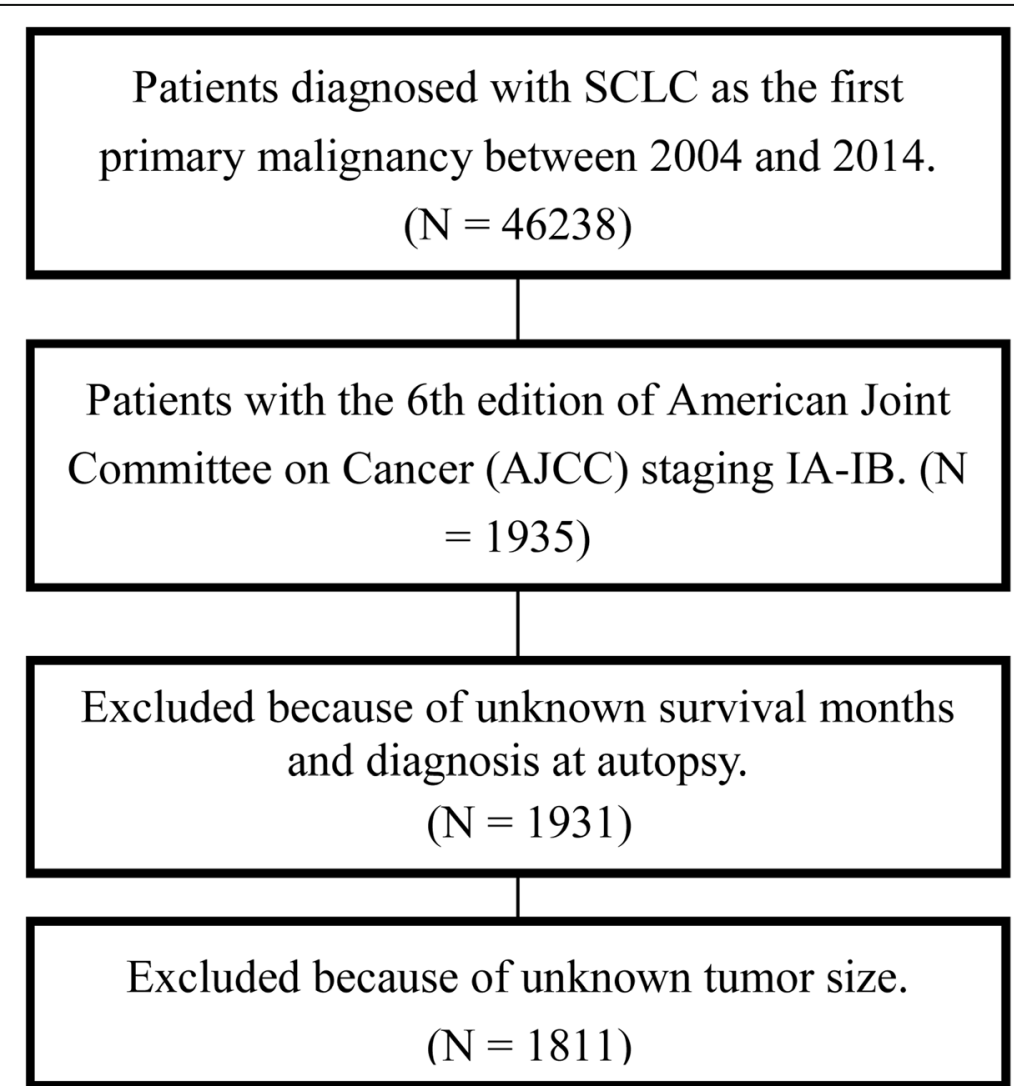

Fig. 1 Flow chart showing the process of patient selection. Patients were selected according to several criteria: (1) stage IA-IB, (2) cases with complete information about survival, follow-up months, and cause of death, (3) cases with known tumor size 
Table 1 One-, three-, and five-year cumulative incidence of mortality in stage I SCLC patients

\begin{tabular}{|c|c|c|c|c|c|c|c|c|c|c|c|c|}
\hline \multirow[t]{2}{*}{ Characteristics } & \multirow[t]{2}{*}{$\mathrm{N}$} & \multirow[t]{2}{*}{$\%$} & \multirow[t]{2}{*}{ Event } & \multirow[t]{2}{*}{$\%$} & \multicolumn{4}{|c|}{ Cancer-specific death } & \multicolumn{4}{|c|}{ Death from other causes } \\
\hline & & & & & 1-year (\%) & 3-year (\%) & 5-year (\%) & $P$ & 1 -year (\%) & 3-year (\%) & 5-year (\%) & $P$ \\
\hline Total & 1811 & & 1221 & & 28.7 & 56.5 & 61.5 & & 5.8 & 11.1 & 13.6 & \\
\hline Age at diagnosis & & & & & & & & $<0.001$ & & & & 0.001 \\
\hline$<60$ years & 342 & 18.9 & 192 & 15.7 & 18.6 & 49.8 & 53.1 & & 5.2 & 7.7 & 8.2 & \\
\hline $60-75$ years & 981 & 54.2 & 640 & 52.4 & 26.1 & 53.5 & 59.5 & & 5.5 & 10.7 & 14.3 & \\
\hline$>75$ years & 488 & 26.9 & 389 & 31.9 & 40.9 & 67.1 & 71.4 & & 6.9 & 14.2 & 15.9 & \\
\hline Gender & & & & & & & & 0.574 & & & & 0.016 \\
\hline Female & 949 & 52.4 & 619 & 50.7 & 27.3 & 56.3 & 60.5 & & 5.1 & 9.1 & 11.3 & \\
\hline Male & 862 & 47.6 & 602 & 49.3 & 30.1 & 56.7 & 62.6 & & 6.5 & 13.3 & 16.0 & \\
\hline Race & & & & & & & & 0.690 & & & & 0.183 \\
\hline White & 1578 & 87.1 & 1064 & 87.1 & 28.9 & 56.1 & 60.8 & & 5.9 & 11.3 & 13.8 & \\
\hline Black & 167 & 9.2 & 111 & 9.1 & 26.4 & 57.9 & 66.0 & & 5.7 & 7.8 & 9.9 & \\
\hline Others & 66 & 3.7 & 46 & 3.8 & 27.7 & 64.3 & 66.6 & & 3.3 & 15.1 & 17.4 & \\
\hline Anatomic sites & & & & & & & & $<0.001$ & & & & 0.048 \\
\hline Upper & 1025 & 56.6 & 685 & 56.1 & 26.5 & 55.9 & 60.6 & & 5.6 & 11.8 & 14.5 & \\
\hline Middle & 115 & 6.4 & 76 & 6.2 & 22.7 & 51.6 & 55.1 & & 12.5 & 19.8 & 19.8 & \\
\hline Lower & 505 & 27.9 & 336 & 27.5 & 29.4 & 55.7 & 61.8 & & 5.1 & 8.0 & 11.1 & \\
\hline Bronchus/others & 166 & 9.2 & 124 & 10.2 & 43.6 & 66.0 & 70.5 & & 4.5 & 9.9 & 10.8 & \\
\hline Primary tumor location & & & & & & & & 0.221 & & & & 0.682 \\
\hline Left-sided & 793 & 43.8 & 522 & 42.8 & 28.1 & 55.3 & 59.8 & & 6.2 & 12.0 & 14.1 & \\
\hline Right-sided & 1018 & 56.2 & 699 & 57.2 & 29.1 & 57.4 & 62.8 & & 5.5 & 10.4 & 13.1 & \\
\hline Tumor size & & & & & & & & $<0.001$ & & & & 0.030 \\
\hline$\leq 3 \mathrm{~cm}$ & 967 & 53.4 & 602 & 49.3 & 23.7 & 50.6 & 55.0 & & 5.7 & 12.1 & 15.7 & \\
\hline $3-5 \mathrm{~cm}$ & 523 & 28.9 & 379 & 31.0 & 30.5 & 59.6 & 66.1 & & 7.1 & 10.9 & 12.1 & \\
\hline$>5 \mathrm{~cm}$ & 321 & 17.7 & 240 & 19.7 & 40.5 & 68.8 & 73.3 & & 3.9 & 8.5 & 9.5 & \\
\hline Tumor extension & & & & & & & & $<0.001$ & & & & 0.005 \\
\hline Local & 1526 & 84.3 & 1020 & 83.5 & 27.9 & 55.4 & 59.7 & & 6.3 & 11.8 & 14.6 & \\
\hline Regional & 285 & 15.7 & 201 & 16.5 & 32.5 & 62.2 & 71.3 & & 3.3 & 7.3 & 8.0 & \\
\hline Grading & & & & & & & & 0.038 & & & & 0.375 \\
\hline Good or moderate & 34 & 1.9 & 18 & 1.5 & 16.3 & 36.6 & 41.3 & & 3.1 & 6.8 & 16.7 & \\
\hline Poor & 343 & 18.9 & 207 & 17.0 & 26.3 & 51.3 & 54.1 & & 4.9 & 11.8 & 13.3 & \\
\hline Undifferentiated & 439 & 24.2 & 318 & 26.0 & 26.5 & 58.0 & 65.0 & & 5.6 & 8.4 & 11.6 & \\
\hline NOS & 995 & 54.9 & 678 & 55.5 & 30.8 & 58.1 & 62.9 & & 6.3 & 12.3 & 14.5 & \\
\hline Surgery & & & & & & & & $<0.001$ & & & & 0.593 \\
\hline Yes & 457 & 25.2 & 214 & 17.5 & 11.2 & 35.6 & 40.4 & & 4.6 & 9.5 & 12.0 & \\
\hline No & 1354 & 74.8 & 1007 & 82.5 & 34.5 & 63.4 & 68.5 & & 6.2 & 11.6 & 14.1 & \\
\hline Chemotherapy & & & & & & & & $<0.001$ & & & & $<0.001$ \\
\hline Yes & 1217 & 67.2 & 783 & 64.1 & 23.8 & 54.0 & 60.0 & & 3.6 & 8.6 & 11.2 & \\
\hline No & 594 & 32.8 & 438 & 35.9 & 38.7 & 61.8 & 64.6 & & 10.4 & 16.3 & 18.6 & \\
\hline Radiotherapy & & & & & & & & $<0.001$ & & & & 0.773 \\
\hline Yes & 929 & 51.3 & 595 & 48.7 & 21.7 & 52.1 & 58.8 & & 4.1 & 10.3 & 13.3 & \\
\hline No & 882 & 48.7 & 626 & 51.3 & 36.0 & 61.2 & 64.4 & & 7.6 & 12.0 & 13.8 & \\
\hline
\end{tabular}




\section{Probability of death}

The cumulative incidence function curves are plotted in Fig. 2. The one-, three-, and five-year estimates of the cumulative incidence of mortality according to the age at diagnosis, gender, race, anatomic sites, laterality, tumor size, tumor extension, grading, and treatment are summarized in Table 1. The five-year cumulative incidence of mortalities resulting from specific causes and other causes was 61.5 and $13.6 \%$, respectively. Patients with the characteristics of big tumor size, regional tumor extension, older age, and no surgery, chemotherapy, and radiotherapy were associated with high cause-specific death probabilities. Patients aged more than 75 years had the highest probability of death resulting from specific causes (71.4\%). The cumulative incidence of causespecific death for patients who did not undergo surgery was as low as $40.4 \%$. As for the patients who did not receive chemotherapy and radiotherapy, their cumulative incidence of cause-specific death was 64.6 and $64.4 \%$, respectively.

Considering the non-linear effect of age and tumor size, we used restricted cubic splines to flexibly model continuous variables. We conducted the joint test to see whether the group of coefficients as a whole was statistically significant or not $(P<0.001)$. As the results of competing risk model displayed on Table 2, tumor size, extent of tumor, laterality of tumor, surgery, and radiotherapy could strongly predict cancer-specific death. Patients who underwent surgery or radiotherapy had a lower cause-specific mortality, with a subdistribution hazard ratios (sdHR) of 0.370 (95\%CI 0.304-0.450) and 0.553 (95\%CI 0.477-0.641), respectively. Patients with regional tumor extension were more likely to die of their disease, with an sdHR of 1.434 (95\%CI 1.216-1.693), when compared with local extension. Additionally, rightsided and larger tumor size were also associated with worse cancer-specific outcomes. For those patients who died from other causes, age, male, local extension, and patients without chemotherapy had a more aggressive impact, with a higher sdHR.

\section{Nomogram}

The nomogram built on the basis of Fine and Gray's model is shown in Fig. 3. The nomogram was used to find the corresponding score on the points row above the graph for each variable included in the model. All the assigned scores of the variables were added to obtain the total score, and then, a straight line was drawn to the bottom of the graph to estimate the probability of death.

\section{Model performance}

The Harrell C index [5] was applied to indicate the discrimination, and a calibration plot obtained using the method provided by Gray [2], which was adopted to evaluate calibration. Discrimination, as measured by the 1000 resample bootstrap-corrected $C$ index, was 0.696 (95\% CI: 0.688-0.705) for the cancer-specific death and 0.672 (95\% CI: 0.650-0.694) for other causes resulting in death. The calibration plot (Fig. 4) showed a high consistency between the predicted and the observed events.

\section{Discussion}

In this study, we assessed the cumulative incidence of mortality resulting from different causes in stage I SCLC patients, who were a part of a large cohort considered in the SEER database. At the same time, we constructed a proportional sub-distribution model and a competing risk nomogram with variables to investigate the three- and five-year cause-specific mortality.

Previous study [11-16] showed that the five-year cumulative incidence rate in patients diagnosed with stage I SCLC who were instructed to undergo surgery was from 40 to $60 \%$. A retrospective analysis from the SEER database showed that patients with stage I SCLC who underwent lobectomy had a higher 5-year survival of $50.3 \%$ [17]. In our study, the five-year cumulative incidence rates of cause-specific and other cause-related mortality were 61.5 and $13.6 \%$, respectively, indicating that SCLC had a high mortality rate and poor prognosis. However, many patients died from other diseases despite the poor prognosis. With an increase in the age and the tumor size, the cumulative incidence of death resulting from all the causes gradually increased. The treatment of SCLC, including surgery, chemotherapy, and radiotherapy, diminished the cumulative incidence of mortality of all the causes. The regional extent of a tumor statistically increased the cumulative incidence, which indicated that the treatment of the limited early stage of cancer was beneficial to the patients' prognosis. For example, a 70 years patient with tumor size of $4 \mathrm{~cm}$ and regional extent of tumor, receiving surgery and radiotherapy has an estimate of 3-year and 5-year probability of death due to lung cancer of 33.7 and $38.1 \%$, respectively.

According to the present competing risk model, the predictors of cause-specific death for stage I SCLC included tumor size, extent of tumor, surgery, and radiotherapy. There was a high probability in patients with the characteristics of the regional extent of tumor, large tumor size, no surgery, or radiotherapy to die of SCLC. Gender did not affect the causespecific mortality, but the male patients were more prone to dying from other causes. Age affected other cause-related SCLC mortality. Hence, it is important 


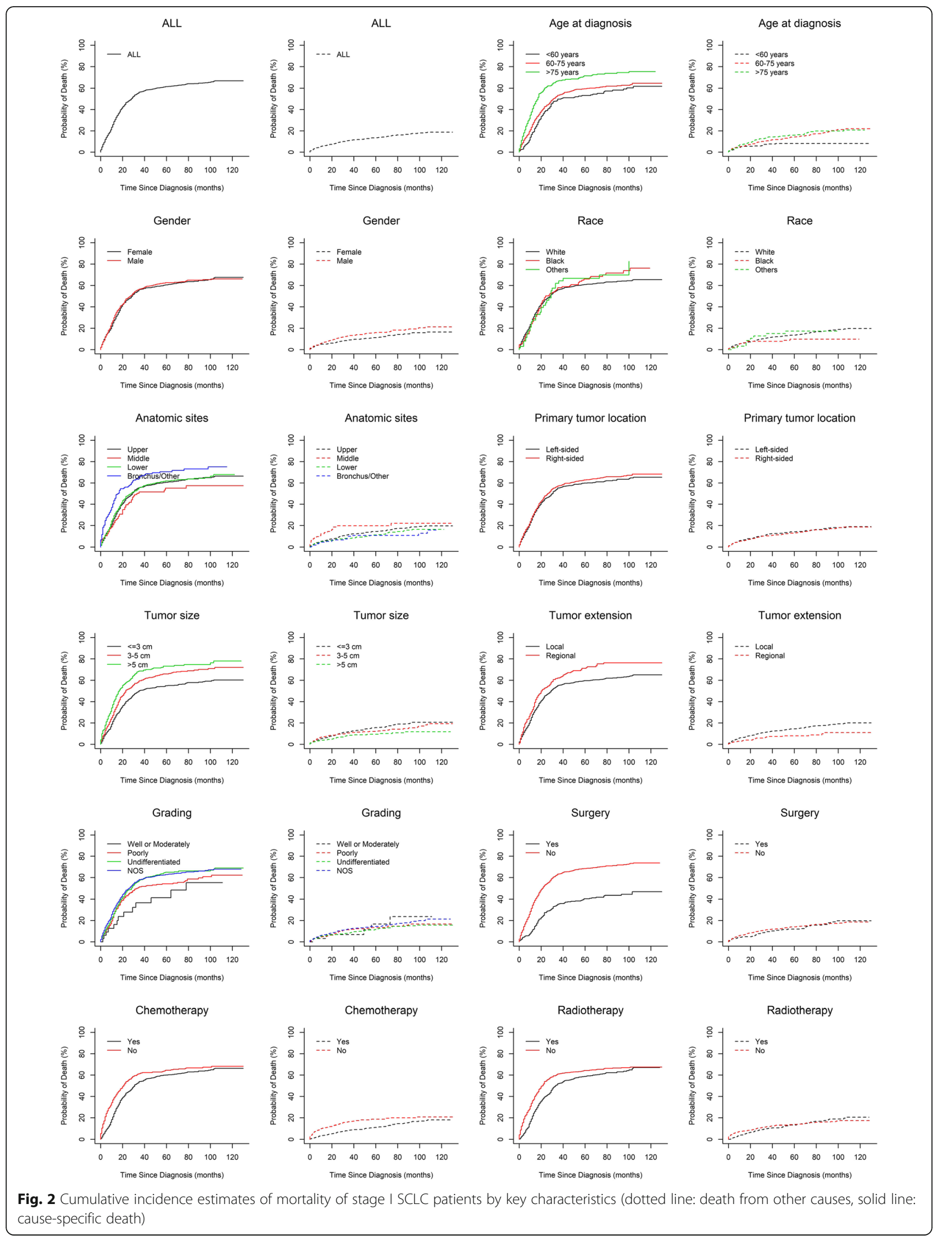


Table 2 Proportional Subdistribution Hazard Models of Probabilities of Cancer-Specific Death and Death from Other Causes for Patients with Stage I SCLC

\begin{tabular}{|c|c|c|c|c|c|c|}
\hline \multirow[t]{2}{*}{ Characteristics } & \multicolumn{3}{|c|}{ Cancer-Specific Death } & \multicolumn{3}{|c|}{ Death from Other Causes } \\
\hline & Coefficient & sd $\mathrm{HR}(95 \% \mathrm{Cl})$ & P & Coefficient & ${ }_{\text {sd }} \mathrm{HR}(95 \% \mathrm{Cl})$ & P \\
\hline Age & 0.011 & $1.010(0.997-1.024)$ & 0.130 & 0.056 & $1.057(1.010-1.105)$ & 0.015 \\
\hline Age' $^{\prime}$ & 0.015 & $1.015(0.999-1.031)$ & 0.065 & -0.050 & $0.951(0.911-0.993)$ & 0.024 \\
\hline Tumor size & 0.118 & $1.125(1.013-1.249)$ & 0.027 & -0.106 & $0.899(0.746-1.083)$ & 0.260 \\
\hline Tumor size' & -0.071 & $0.931(0.806-1.076)$ & 0.340 & 0.044 & $1.045(0.780-1.399)$ & 0.770 \\
\hline Male & -0.024 & $0.976(0.858-1.110)$ & 0.720 & 0.332 & $1.393(1.071-1.811)$ & 0.013 \\
\hline \multicolumn{7}{|l|}{ Race } \\
\hline Black & -0.022 & $0.978(0.805-1.187)$ & 0.830 & -0.454 & $0.634(0.368-1.094)$ & 0.100 \\
\hline Others & -0.159 & $0.853(0.591-1.231)$ & 0.400 & -0.028 & $0.972(0.490-1.929)$ & 0.940 \\
\hline \multicolumn{7}{|l|}{ Anatomic sites } \\
\hline Middle & -0.248 & $0.780(0.580-1.050)$ & 0.100 & 0.424 & $1.528(0.937-2.492)$ & 0.089 \\
\hline Lower & 0.011 & $1.010(0.874-1.168)$ & 0.880 & -0.256 & $0.774(0.564-1.060)$ & 0.110 \\
\hline Bronchus/Other & 0.256 & $1.291(1.018-1.636)$ & 0.034 & -0.192 & $0.825(0.492-1.382)$ & 0.470 \\
\hline Right-sided & 0.149 & $1.160(1.015-1.324)$ & 0.028 & -0.132 & $0.876(0.669-1.147)$ & 0.340 \\
\hline Regional & 0.361 & $1.434(1.216-1.693)$ & $<0.001$ & -0.516 & $0.597(0.381-0.934)$ & 0.024 \\
\hline \multicolumn{7}{|l|}{ Grading } \\
\hline Poorly & 0.171 & $1.186(0.701-2.006)$ & 0.520 & -0.213 & $0.807(0.317-2.053)$ & 0.650 \\
\hline Undifferentiated & 0.229 & $1.257(0.748-2.111)$ & 0.390 & -0.353 & $0.702(0.277-1.779)$ & 0.460 \\
\hline NOS & 0.156 & $1.168(0.698-1.955)$ & 0.550 & -0.108 & $0.897(0.356-2.259)$ & 0.820 \\
\hline Surgery & -0.992 & $0.370(0.304-0.450)$ & $<0.001$ & -0.162 & $0.850(0.590-1.223)$ & 0.380 \\
\hline Chemotherapy & -0.064 & $0.937(0.801-1.096)$ & 0.420 & -0.582 & $0.558(0.411-0.758)$ & $<0.001$ \\
\hline Radiotherapy & -0.592 & $0.553(0.477-0.641)$ & $<0.001$ & 0.182 & $1.199(0.885-1.625)$ & 0.240 \\
\hline
\end{tabular}

Note: Age' and Tumor size' are constructed spline variables (when $\mathrm{k}=3$ )

to take actions to prevent older patients from dying from other diseases irrespective of the SCLC treatment. We did not find any significant effects of race and laterality on cause-specific death and death from other causes. Anatomic sites and grading were only significant in the cases of cause-specific death. Wang et al. [18] developed a nomogram prognostic model for SCLC patients and validated the model using an independent patient cohort. Their nomogram performs better than earlier models, including those using AJCC staging. However, because of lacking the Stage I SCLC competing risk analyses in their model, we cannot compare the results between Wang's model and our model in this study.

Patients diagnosed with SCLC without any lymph node metastasis at a very early stage may undergo surgical resection of the lesion as the initial treatment procedure. According to the National Comprehensive Cancer Network guidelines, postoperative chemotherapy is recommended for stage I SCLC rather than radiation. Our study showed that surgery could effectively reduce the number of cancer-specific deaths and that the one-year cumulative incidence dropped from 34.5 to $11.2 \%$. Like surgery, chemotherapy and radiotherapy improved the one-year survival rate. It is necessary to consider radiation before or after surgery, and this needs more validation. As SCLC is characterized by rapid growth, high invasiveness, and early metastasis, the five-year cumulative incidence was relatively high irrespective of the form of treatment. Our results indicated that treatment did not benefit the five-year survival rate. Therefore, early diagnosis and treatment are very critical and can markedly improve the one-year survival rate.

It is undeniable that our prediction model has some limitations. First, approximately $27 \%$ of the patients in our study were diagnosed during 2012-2014, which resulted in relatively short follow-up time. We could expect that longer follow-up time may help to improve the accuracy of model prediction. Second, several treatment-related factors weren't included in the model, such as the plans of chemotherapy, number of cycles, the doses and methods of radiotherapy and the follow up treatment after recurrence. These 


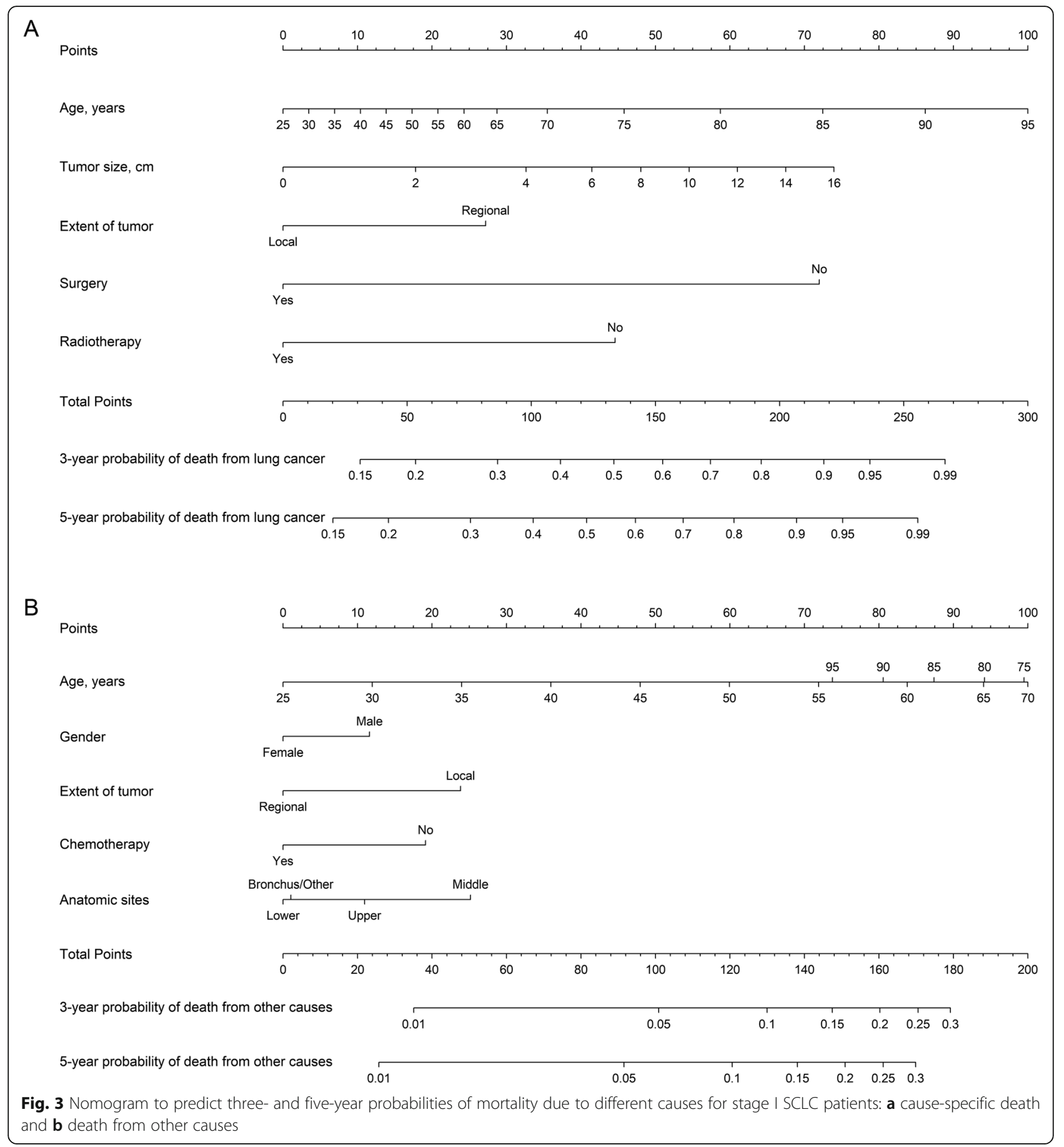

factors can also influence the prognosis. Third, our model only provides a reference to clinical doctors. More complicated clinical factors will also be taken into account in their treatment decisions. Fourth, the comorbidity was a significant factor when physicians deciding treatment strategies. It was indeed a limitation that we established a prognostic model without comorbidity information. But we considered other vital clinical characters which could be obtained in
SEER database with large sample and we believed this model could also providing valuable implications in clinical practice for stage I SCLC patients.

\section{Conclusions}

The cumulative incidence of mortality due to specific causes and other causes in stage I SCLC patients was calculated using a SEER database analysis. We also constructed the competing risk regression model for stage I 


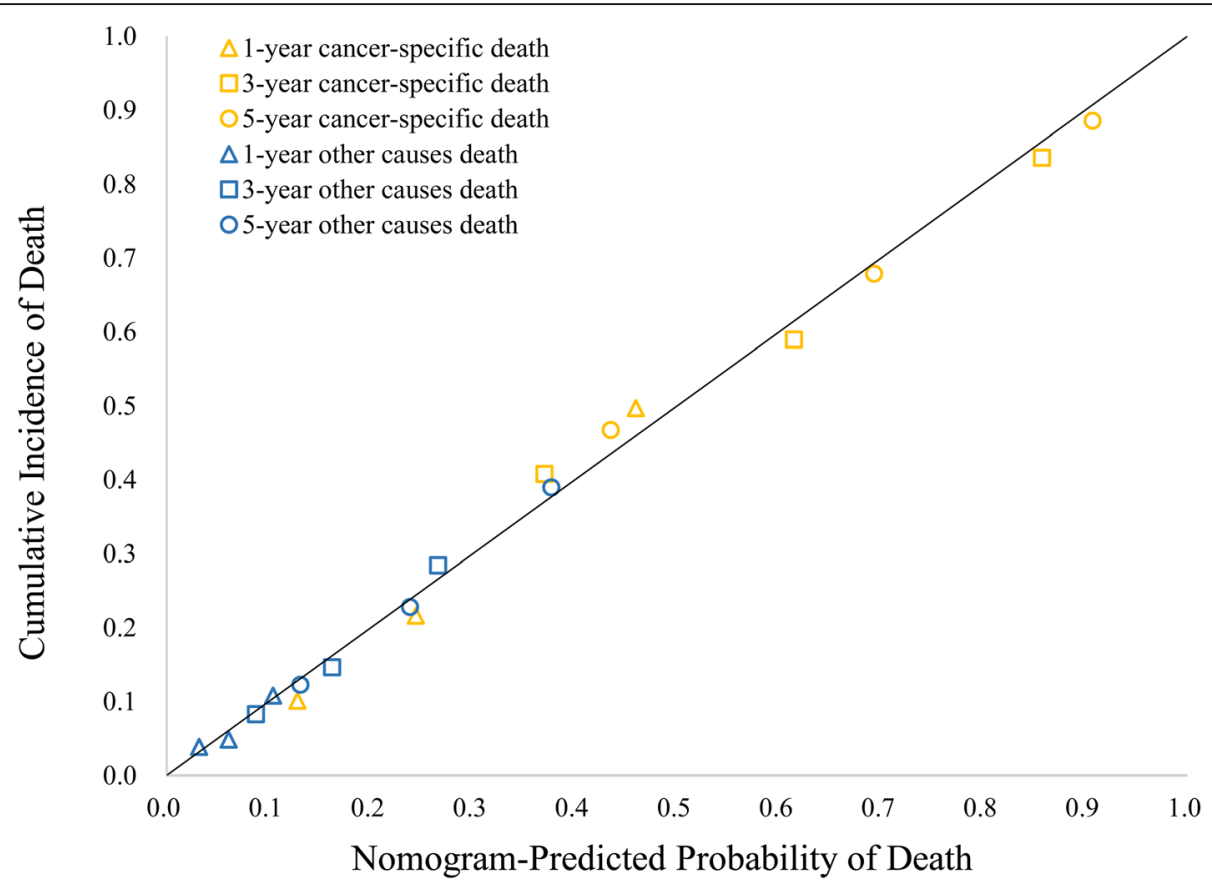

Fig. 4 Calibration plot indicating the performance of the nomogram

SCLC and a competing risk nomogram to predict the three- and five-year cause-specific mortality individually. The nomogram could predict the prognosis conveniently and directly for stage I SCLC patients and help clinicians to make critical treatment decisions and choose appropriate strategies.

\section{Supplementary information}

Supplementary information accompanies this paper at https://doi.org/10. 1186/s12885-020-07271-9.

\section{Additional file 1.}

\section{Abbreviations}

SCLC: Small-cell lung cancer; CIFs: Cumulative incidence functions; NCCN: National Comprehensive Cancer Network; SEER: Surveillance, Epidemiology, and End Results; sdHR: Sub-distribution hazard ratio; COPD: Chronic obstructive pulmonary disease; NOS: Not otherwise specified; Cl: Confidence interval

\section{Acknowledgments}

The authors acknowledge the efforts of the SEER program in the creation of the SEER database.

\section{Authors' contributions}

ZP. W and J.Z Conceived and designed of the research. JJ. L and QW. Z carried out data acquisition analysis and interpretation. JJ. L and XH. Z drafted and revised the manuscript. TT. A, MN. W, YY. W and ML. Z provided assistance for the interpretation of the results. J. Z, B. J, X. Y and HX. C provided assistance for data acquisition, data analysis and statistical analysis. Z. D, JJ. W, YJ. C and XY. Z collected the background information. All the authors have read and approved the content of the manuscript.

\section{Funding}

This work was financially supported by the Science Foundation of Peking University Cancer Hospital(18-02); Beijing Municipal Administration of Hospitals Incubating Program (PX2019038); Science Foundation of Peking University Cancer Hospital (2017-18).

\section{Availability of data and materials}

Limited Use Agreement for Surveillance, Epidemiology, and End Results (SEER) Program (https://seer.cancer.gov) SEER*Stat Database: accession number (15586-Nov2016). The data can be used publicly.

\section{Ethics approval and consent to participate}

The study was exempted from ethical review by the Beijing Cancer Hospital. We obtained the data agreement and downloaded the files directly from the SEER website in accordance with SEER requirements. The reference number was 15586-Nov2016.

\section{Consent for publication}

Not applicable.

\section{Competing interests}

The authors declare that they have no competing interests.

\section{Author details}

${ }^{1}$ Key Laboratory of Carcinogenesis and Translational Research (Ministry of Education/Beijing), Department of Thoracic Medical Oncology, Peking University Cancer Hospital \& Institute, 52 Fucheng Road, Haidian District, Beijing 100142, China. ${ }^{2}$ Department of Epidemiology and Biostatistics, School of Public Health, Peking University, Beijing, China.

Received: 19 September 2019 Accepted: 7 August 2020

Published online: 24 August 2020

\section{References}

1. Lu T, Yang XD, Huang YW, Zhao MN, Li M, Ma K, Yin JC, Zhan C, Wang Q. Trends in the incidence, treatment, and survival of patients with lung cancer in the last four decades. Cancer Manag Res. 2019;11:943-53. 
2. National Comprehensive Cancer Network. (NCCN) Clinical Practice Guide lines in Oncology. Small Cell Lung Cancer (V2.2018). Available at https:// www.nccn.org/professionals/physician_gls/pdf/sclc.pdf.

3. Gray RJ. A class of k-sample tests for comparing the cumulative incidence of a competing risk. Ann Stat. 1988;16:1141-54.

4. Fine JP, Gray RJ. A proportional hazards model for the sub-distribution of a competing risk. J Am Stat Assoc. 1999;94:496-509.

5. Harrel FE. Regression Modeling strategies: general aspects of fitting regression models. New York, NY: Springer; 2001.

6. Harrell F. Regression modeling strategies: with applications to linear models, logistic and ordinal regression, and survival analysis. Springer Series in Statistics: Springer; 2015

7. Wolbers M, Koller MT, Witteman JC, Steyerberg EW. Prognostic models with competing risks: methods and application to coronary risk prediction. Epidemiology. 2009:20:555-61.

8. Harrell FJ, Lee KL, Mark DB. Multivariable prognostic models: issues in developing models, evaluating assumptions and adequacy, and measuring and reducing errors. Stat Med. 1996;15(4):361-87.

9. Gray B. cmprsk: Sub-distribution Analysis of Competing Risks. R package version 2.2-7; 2014

10. de Liesbeth C. Wreede, Marta Fiocco, Hein putter. Mstate: an R package for the analysis of competing risks and multi-state models. J Stat Slftw. 2011; 38(7):1-30.

11. Harrell FE. rms: Regression Modeling Strategies. R package version 5.1-2; 2018.

12. Schreiber D, Rineer J, Weedon J, Vongtama D, Wortham A, Kim A, Han P, Choi K, Rotman M. Survival outcomes with the use of surgery in limitedstage small cell lung cancer: should its role be re-evaluated? CANCER-AM CANCER SOC. 2010;116(5):1350-7.

13. Brock MV, Hooker CM, Syphard JE, Westra W, Xu L, Alberg AJ, Mason D, Baylin SB, Herman JG, Yung RC, et al. Surgical resection of limited disease small cell lung cancer in the new era of platinum chemotherapy: its time has come. J Thorac Cardiovasc Surg. 2005;129(1):64-72.

14. Lim E, Belcher E, Yap YK, Nicholson AG, Goldstraw P. The role of surgery in the treatment of limited disease small cell lung cancer: time to reevaluate. $J$ Thorac Oncol. 2008;3(11):1267-71.

15. Shields TW, Higgins GJ, Matthews MJ, Keehn RJ. Surgical resection in the management of small cell carcinoma of the lung. J Thorac Cardiovasc Surg. 1982;84(4):481-8.

16. Yu JB, Decker RH, Detterbeck FC, Wilson LD. Surveillance epidemiology and end results evaluation of the role of surgery for stage I small cell lung cancer. J Thorac Oncol. 2010;5(2):215-9.

17. Schneider BJ, Saxena A, Downey RJ. Surgery for early-stage small cell lung cancer. J Natl Compr Cancer Netw. 2011;9(10):1132-9.

18. Wang S, Yang L, Ci B, Maclean M, Gerber DE, Xiao G, Xie Y. Development and validation of a Nomogram prognostic model for SCLC patients. J Thorac Oncol. 2018;13(9):1338-48.

\section{Publisher's Note}

Springer Nature remains neutral with regard to jurisdictional claims in published maps and institutional affiliations.

Ready to submit your research? Choose BMC and benefit from:

- fast, convenient online submission

- thorough peer review by experienced researchers in your field

- rapid publication on acceptance

- support for research data, including large and complex data types

- gold Open Access which fosters wider collaboration and increased citations

- maximum visibility for your research: over $100 \mathrm{M}$ website views per year

At BMC, research is always in progress.

Learn more biomedcentral.com/submissions 